\title{
Recent progress in bone imaging for osteoporosis research
}

\author{
Masako Ito \\ Department of Radiology, Nagasaki University School of Medicine \\ 1-7-1 Sakamoto, Nagasaki 852-8501 JAPAN \\ Phone: 81-95-819-7434
}

\section{Abstract}

Advances in bone imaging techniques have provided tools for analyzing bone structure at the macro-, micro- and nano-level. Quantitative assessment of macrostructure can be achieved using dual X-ray absorptiometry (DXA) and quantitative computed tomography (QCT), particularly volumetric quantitative CT (vQCT). In vivo quantitative techniques for assessing the microstructure of trabecular bone non-invasively and non-destructively include high-resolution CT (HR-CT) and high-resolution magnetic resonance (HR-MR). Compared with MR imaging, CT-based techniques have the advantage of directly visualizing the bone in the axial skeleton, with high spatial resolution, but the disadvantage of delivering a considerable radiation dose.

Micro-CT $(\mu \mathrm{CT})$, which provides a higher resolution of the microstructure and is principally applicable in vitro, has undergone technolgical advances such that it is now able to elucidate the physiological skeletal change mechanisms associated with aging and 
determine the effects of therapeutic intervention on the bone microstructure. In particular, synchrotron $\mu \mathrm{CT}(\mathrm{SR}-\mathrm{CT})$ provides a more detailed view of trabecular structure at the nano-level.

For the assessment of hip geometry, DXA-based Hip Structure Analysis (HSA) and CT-based HSA have been developed. DXA-based HSA is a convenient tool for analyzing biomechanical properties and for assuming cross-sectional hip geometry based on two-dimensional (2D) data, while CT-based HSA provides these parameters three-dimensionally in robust relationship with biomechanical properties, at the cost of greater radiation exposure and the lengthy time required for the analytical procedure.

Further progress in bone imaging technology is promising to bring new aspects of bone structure in relation to bone strength to light, and to establish a means for analyzing bone structural properties in the every day clinical setting.

Key word

computed tomography, micro-CT, magnetic resonance, microstructure, geometry, finite element model 
Introduction

Non-invasive techniques, such as computed tomography (CT) and magnetic resonance imaging (MRI), provide information on structural properties much more effectively than conventional radiography or bone densitometry. It is known that bone mineral density (BMD) only partly explains bone strength. Thanks to quantitative assessment of macro- and micro- structural properties, the ability to estimate bone biomechanical properties has been improved. Besides determining bone strength, bone imaging provides a great deal of new information on the physiological architectural response to aging, weightlessness, and certain other disorders, as well as the response to pharmacological intervention.

Compared with MRI, CT has the advantage of direct visualization of the bone with high spatial resolution, but it also has the disadvantage of delivering a considerable radiation dose if applied to the central skeleton. CT devices cover a wide range of spatial resolution; clinical whole body CT scanners provide an in-plane spatial resolution of less than $200 \mu \mathrm{m}$, with a slice thickness of approximately $500 \mu \mathrm{m}$; in vitro micro-CT $(\mu \mathrm{CT})$ is capable of bone imaging with isotopic spatial resolutions of approximately $10 \mu \mathrm{m}$; synchrotron $\mu \mathrm{CT}$ systems achieve spatial resolutions of less than $1 \mu \mathrm{m}$. The introduction of micro-CT has 
facilitated significant progress in elucidating the mechanisms of the physiological skeletal changes which occur with aging and helped clarify the effects of various interventions on bone microstructure, while synchrotron CT has enabled more precise micro- to nano-structure as well as information on the degree of mineralization.

Clinical application of bone imaging for the in vivo assessment of structural properties is challenging. For the in vivo assessment of microstructure, clinical high resolution $\mathrm{CT}$ is employed to derive information similar to that for micro-CT, even though it falls short of the spatial resolution required to visualize the trabecular network. Therefore, in vivo applications of non-destructive and non-invasive techniques are at the forefront of radiological research in osteoporosis.

In this article, I will discuss recent progress in bone imaging in a range from the nano- to macro-structures in order to investigate the structural basis of the skeletal fragility underlying osteoporosis, as well as to assess bone biomechanical properties, focusing mainly on CT. 


\section{Assessment of BMD by QCT}

One advantage of QCT, compared with DXA, is the capacity for separate analysis of the BMD of the trabecular and cortical compartments. QCT also provides real bone density per bone volume $\left(\mathrm{mg} / \mathrm{cm}^{3}\right)$ in the axial as well as the peripheral skeleton. Recently, 3D volume data from the scanning of an entire bone, such as a vertebral body or proximal femur, can be reconstructed to adjust the exact selected region for several serial images in a longitudinal study, which enables a monitoring of successive changes with very good precision.

1. Volumetric Quantitative Computed Tomography (vQCT)

Trabecular bone, particularly in the spine, is metabolically more active and may therefore serve as an early indicator of treatment effect, and vertebral trabecular BMD was demonstrated to have a significant correlation with vertebral fracture ${ }^{1-3)}$. Cortical bone, however, particularly in the hip, may be more useful for estimating hip fracture risk.

Vertebral trabecular BMD bears a significant relationship with menarch or menopause ${ }^{4)}$, anthropometric parameters ${ }^{5)}$, and a racial difference between Japanese and American women has been reported ${ }^{6}$. Even in cases of spondylosis deformans in men, QCT provides an accurate measure of trabecular BMD, with no significant influence of 
osteophytes $^{7)}$.

The International Society of Clinical Densitometry (ISCD) position paper on QCT was published two years $\mathrm{ago}^{8)}$. The recognition of the utility of QCT in clinical studies has been growing world wide.

\section{Peripheral QCT (pQCT)}

The utility and limitations of $\mathrm{pQCT}{ }^{9-10)}$ have been discussed in various reviews. Two interesting results using $\mathrm{PQCT}$ are here taken into consideration.

Using peripheral QCT (Densiscan1000, ScancoMedical), which provides a very high degree of precision (less than $1 \%$ error), a longitudinal study was performed to evaluate the change in trabecular and cortical BMD after hip surgery and subsequent rehabilitation ${ }^{11)}$. Even relatively brief non-weight-bearing periods led to significant bone loss, and 1-1.5 years was required to recover to the baseline BMD. The recovery of the decrease in the cortical region occurred after the recovery in the trabecular and endosteal regions.

To clarify the effects of habitual volleyball exercise on bone in women during the menopausal period, we measured BMD in the lumbar spine, calcaneus and tibia every 12 months for 2 years and estimated the factors related to the baseline values and annual loss rates. It was concluded that the total number of years participating in 
exercise activity during adulthood has a positive effect on lumbar and calcaneus BMD and the cortical structure of the tibia. Habitual volleyball exercise did not alleviate menopause-related bone loss in the lumbar spine ${ }^{12)}$.

II. Assessment of the trabecular and cortical microstructure

The goals of quantification of trabecular bone architecture in the assessment of osteoporosis are as follows: 1 ) investigating the mechanisms of physiological bone change, 2) determining the prevalence and/or incidence of osteoporotic fractures, and 3) monitoring the response of the trabecular architecture to pharmacological treatment.

\section{Micro-CT $(\mu-C T)$}

The micro-CT system has been demonstrated to be the first device able to non-destructively reveal the "real" trabecular architecture, and is an x-ray-based technique which provides 3D images of very high isotropic spatial resolutions $\left(6 \mu \mathrm{m}^{3}<\right)$. Since micro-CT allows the depiction of individual trabeculae and enables the full characterization of the trabecular network, many investigators have used it to study the trabecular network at different skeletal sites, in direct relation to biomechanical properties or as a "gold standard" for evaluating other 
techniques, although most of the micro-CT studies are limited to ex vivo investigations.

Most of the metric parameters, such as the bone surface area (BS), bone volume (BV), total volume (TV), bone volume fraction (BV/TV), trabecular thickness (Tb.Th) and trabecular separation (Tb.Sp), are directly determined using methods such as trianglulation, direct voxel counting or distance transformation. The trabecular number (Tb.N) is calculated by taking the inverse of the mean distance between the middle axes of the trabeculae.

In addition to the metric parameters, non-metric parameters are calculated to obtain the 3D nature of the trabecular bone samples (Fig. 1). The structure model index (SMI) ${ }^{13)}$ is a parameter used to estimate the plate-rod characteristics of the structure. The geometrical degree of anisotropy (DA) ${ }^{14)}$ is a parameter of trabecular orientation, and is defined as the ratio between the maximal and minimal radii of the mean intercept length (MIL) ellipsoid. Connectivity is calculated using the Euler method of Odgaard and Gundersen (Euler's number) ${ }^{15)}$ (Fig. 1). Using micro-CT, bone samples from several animal models were non-destructively used to investigate pathophysiological change mechanisms ${ }^{16,17)}$, bone fragility ${ }^{18,19)}$ and the response to osteoporosis

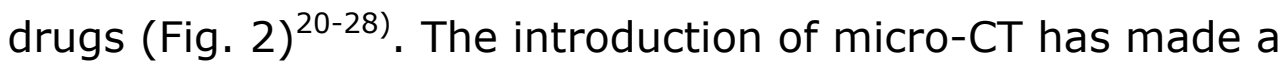


contribution to the field of bone cell biology be enabling the visualization of various phenomena ${ }^{29-32)}$.

\section{Synchrotron CT (SR-CT)}

Synchrotron radiation (SR) provides an intense, laser-like collimated light in a range from infrared to hard X-rays, and also provides an X-ray source for high-resolution micro-CT. SR offers a continuous energy spectrum with a high degree of photon flux and monochromaticity of the beam. Because of the characteristics of the SR white beam, it precisely indicates the different densities according to the level of mineralization and reveals the ultra-structure of the bone. The images in Fig. 3 were scanned at the synchrotron radiation facility known as Super Photon ring-8GeV (SPring-8) ${ }^{33)}$.

SR-CT is applicable to the visualization of subchondral bone in the study of osteoarthrits ${ }^{34)}$, as well as the quantification of the ultra-structure and degree of bone mineralization in bone tissue resulting from osteoporosis ${ }^{35,36)}$ and other pathologies. 3D analysis of the ultra-structure, such as the canal network and the osteocyte lacunar system using SR-CT, revealed microcrack initiation and propagation along with their effects on bone mechanics ${ }^{37)}$. 
3. Magnetic Resonance Imaging (MRI)

MRI is a noninvasive imaging modality that does not require any ionizing radiation, which is a great advantage over CT. On the other hand, in contrast to CT, MRI acquisition takes considerably more time and is technically demanding. In high spatial resolution MRI of bone structures, the dark network (signal void) represents the trabecular bone network and the higher-intensity background represents the marrow in the trabecular spaces. The appearance of the trabecular network is influenced by the imaging sequences; gradient echo sequences, longer time of echo (TE) and higher field strength increase the susceptibility to artifacts and consequently an overestimation of trabecular thickness.

Most in-vivo studies have been performed at sites in the peripheral skeleton, such as the distal radius, distal tibia and the calcaneus, since these regions exhibit a high signal-to-noise $(s / n)$ ratio due to a high number of trabeculae and a high degree of fat-marrow contrast, and are easily accessible with small coils.

Moreover, due to newly developed surface coils and suitable acquisition pulse sequences, in vivo high-resolution trabecular bone images at the proximal femur, with a spatial resolution of $234 \mu \mathrm{m} \times 234$ $\mu \mathrm{m} \times 1500 \mu \mathrm{m}$, have recently been achieved ${ }^{38)}$. Besides the gradient echo sequences obtained using the spin-echo sequence (3D FLASH: 
fast large-angle spin-echo), 3D high-resolution microstructure images of the distal radius are obtained in vivo, which is sometimes called a "virtual bone biopsy" ${ }^{39)}$. The parameters derived using these sequences effectively discriminate between subjects with and without fracture.

Furthermore, for the purpose of drug effect monitoring, the usefulness of MR imaging of trabecular microstructure has been demonstrated in a clinical trial of nasal calcitonin ${ }^{40)}$. In a study of osteoarthritis using different sequences of MRI, the microstructure in subchondral bone as well as articular cartilage were demonstrated and quantified (Fig. 4).

\section{High resolution $\mathrm{pQCT}$}

One pQCT device, XtremeCT (Scanco, Switzerland), depicts in vivo bone microstructure with an isotopic voxel size of $82 \mu \mathrm{m}^{3}$, as well as BMD in the forearm and the tibia. Peripheral trabecular structure assessment has been shown to be useful in differentiating women with an osteoporotic fracture history from controls better than DXA in the hip and spine ${ }^{41)}$. This high-resolution PQCT also demonstrated the different patterns of bone loss in men and women ${ }^{42)}$.

Furthermore, there is currently an interest in conducting analyses of the cortical microstructure. XtremeCT has the capacity to demonstrate 
cortical porosity and determine the different effects of osteoporosis drugs on cortical microstructure ${ }^{43,44)}$, even though the spatial resolution is still limited with regard to revealing the small pores.

\section{Clinical CT Applications}

High-resolution CT for clinical use enables a clear depiction of trabecular and cortical morphology. However, given the typical dimensions of the trabeculae $(100-400 \mu \mathrm{m})$ and trabecular spaces $(200-2000 \mu \mathrm{m})$, this resolution is still at the boundary of being able to determine the trabecular network. Previously, instead of a direct measurement of the structural parameters, analysis of the trabecular bone pattern (texture) was performed using clinical CT. Using texture analysis, characteristics of the trabecular architecture may be extracted without requiring stringent segmentation between the individual trabeculae. In our study using run-length analysis as one of texture analyses ${ }^{45,46)}$, trabecular structure parameters were more effectively distinguished between cases of fracture and non-fracture controls than BMD measurements with DXA, especially in older subjects.

Recently, we developed a CT-system visualizing and analyzing the trabecular microstructure network in vivo. Because the current spatial resolutions in clinical CT and MR are not sufficiently high to depict individual trabeculae, partial volume effects cannot be avoided in 
trabecular bone. Threrefore, the corresponding trabecular bone parameters computed from these imaging modalities are usually called apparent (app.) measures: app. BV/TV, app. Tb.Th, app. Tb.Sp, and app. Tb.N.

We visualized the trabecular microstructure by applying multidetector row CT (MDCT) and evaluated (Fig. 5) in 82 postmenopausal women (55-76 years old), including 39 women with and 43 without a recent vertebral fracture. The microstructure indices obtained by MDCT scanning revealed a higher relative risk for vertebral fracture (Odds ratio: 16.0 for SMI, 13.6 for app. BV/TV, and 13.1 for Euler's number) than did spinal BMD obtained by DXA (Odds ratio: 4.8). MDCT provided volumetric BMD data, which had a higher diagnostic value (Odds ratio: 12.7 ) than did DXA. We concluded that the microstructure parameters obtained by MDCT, together with the volumetric BMD, demonstrated better diagnostic performance for assessing fracture risk than DXA measurement ${ }^{47)}$.

Recently, structural parameters of the spine were analyzed in a longitudinal in vivo study of PTH, and all of the structural variables exhibited significant improvement, with some independence from BMD in the treated group ${ }^{48)}$.

5. Finite element model (FEM) 
FEM is a computer-based simulation of the strains and stresses induced by the mechanical loading of an object, and is an established method in engineering. The aim of FEM is to better predict the load conditions that lead to stress distribution and thus in turn to improve fracture prediction. Currently, the models are typically derived from volumetric QCT data, which are converted into voxel finite element models to yield a measure of bone strength ${ }^{49)}$.

Using a micro-FEM on the basis of micro-CT data, in an ovariectomized rat model we analyzed, how the trabecular and cortical components contribute to the mechanical properties (Figs. 6, 7) ${ }^{50)}$. By applying FEM to clinical studies, the effect of medical treatment on vertebral strength can be predicted, and areas where an alteration in bone strength has occurred can be determined ${ }^{51)}$.

\section{Assessment of hip geometry}

Risk factors for hip fracture include low BMD, local geometrical vulnerability to loading as well as a high incidence of falls. A precise analysis of hip geometry and BMD is important for predicting the risk of hip fracture, and bone imaging is again helpful for this purpose.

\section{DXA-based Hip Structure Analysis (HSA)}

The Hip Structural Analysis (HSA) program determines not only the 
BMD of the hip bone but also the structural geometry of cross-sections traversing the proximal femur at specific locations: 1 ) the Narrow Neck (NN) across the narrowest diameter of the femoral neck, 2)

Intertrochanteric (IT) region, and 3) Shaft ${ }^{52,53)}$.

On the basis of the DXA attenuation profile and assumed cross-sectional area, the HSA algorithm derives the conventional BMD $\left(\mathrm{g} / \mathrm{cm}^{2}\right)$, the outer diameter $(O D, \mathrm{~cm})$, the endocortical diameter (ED, $\mathrm{cm})$, the average cortical thickness (CoTh, $\mathrm{cm}$ ), the total mineralized bone area in the cross-section $\left(\mathrm{CSA}, \mathrm{cm}^{2}\right)$, the cross-sectional moment of inertia $\left(\mathrm{CSMI}, \mathrm{cm}^{4}\right)$ and the section modulus $\left(\mathrm{SM}, \mathrm{cm}^{3}\right) . \mathrm{SM}$ is computed as CSMI/d $d_{\max }$, where $d_{\max }(\mathrm{cm})$ is the maximum distance between the center of the mass (centroid) and the outer cortex. Another biomechanical parameter, the buckling ratio (BR), is estimated as the ratio of the $d_{\max }$ to the estimated average CoTh derived from an annulus model of the cross-section using the measured OD, assuming that a fixed proportion of CSA is in the cortex. CSA and SM are indices of resistance to axial compressive and bending loads, respectively, and BR is an index of susceptibility to local buckling in cases of very thin-walled bone subjected to bending loads.

As an example of the clinical studies of DXA-based HSA, the effect of the bisphosphonate minodronic acid hydrate on the geometry of the proximal femur was analyzed in 103 postmenopausal Japanese patients 
with osteoporosis ${ }^{54)}$. It was found that the bisphosphonate reduced age-related endocortical bone resorption, leading to increased cortical thickness and sustained or enhanced bone strength. Many clinical studies have reported the efficacy of anti-osteoporotic agents. Prospective studies are required to determine whether DXA-based HSA is able to predict future fractures.

\section{CT-based HSA}

The assessment modality of hip geometry using clinical CT is called "CT-based HSA". CT-based HSA with volumetric 3D data acquisition is a promising way to define the exact femoral neck axis and the cross-section of femoral neck, and to obtain a reproducible location of a given volume of interest (VOI) in longitudinal scans.

From the 3D hip data, the axis of the femoral neck is defined and Hip Axis length (HAL) is calculated. On the basis of the femoral neck axis, the cross-sectional image is defined to calculate the total, trabecular and cortical BMD/cross-sectional area (CSA)/bone mass as well as cortical thickness and cortical perimeter (Fig.8). As biomechanical parameters, CSMI, SM and BR are obtained in a similar manner to DXA-based HSA, however, $d_{\max }$ must be defined more precisely than DXA-based HSA. 
The analysis of bone distribution in the mid-femoral neck of 77 proximal cadaver femurs demonstrated that, with aging, the cortical density and thickness in the supero-posterior region decreased, and the cortical thickness in the inferior region increased ${ }^{55)}$. They concluded that as women age, hip fragility increases because under-loading of the supero-posterior cortex leads to atrophic thinning, and this imbalance induces instability of the femoral neck.

Such a detailed analysis of local changes in the geometry of the femoral neck is only feasible by high-resolution 3D CT imaging. We have designed two case-control studies to determine the characteristics of the femoral neck structure associated with femoral neck fracture or trochanteric fracture using clinical CT; the neck fracture study included 20 Japanese women with neck fracture and 20 age-matched control women, and the trochanteric fracture study included 16 Japanese women with trochanteric fracture and 16 age-matched control women. We found women with neck fracture had a significantly longer HAL, lower CSMI, and higher BR, and women with trochanteric fracture had a significantly smaller cortical CSA of the femoral neck ${ }^{56)}$. Therefore, we conclude that clinical CT may be useful for the assessment of the risk of neck and trochanteric fracture. The longitudinal changes in cross-sectional geometry in the femoral neck in Japanese post-menopausal women, were also investigated ${ }^{57)}$. 
Finite element models of QCT scans biomechanically integrates material and geometric information to produce an estimate of whole bone strength that is a better predictor of actual strength than estimators derived from QCT alone ${ }^{58)}$.

Conclusion

In conclusion, the purpose of bone imaging for osteoporosis research is mainly to improve the assessment of skeletal fragility in vivo, and also to elucidate the mechanism underlying various bone disorders using human samples and animal models. Table 1 summarizes the various bone imaging modalities used to evaluate bone quantity and quality. Further research is required to improve the reproducibility, standardization and number of clinical applications. New technological advances may further refine the imaging of osteoporotic bone and assessment of fracture risk.

Acknowledgments

I thank Dr. Kyoji Ikeda (Department of Bone and Joint Disease, National Center for Geriatrics and Gerontology, Obu, Aichi, Japan) for comments on the manuscript. This study was supported in part by a grant for the Promotion of Fundamental Studies in Health Sciences of the National Institute of Biomedical Innovation (NIBIO) of Japan 
(\#06-31 to MI). Pacific Edit reviewed the manuscript before submission. 


\section{References}

1. Ito M, Hayshi K, Kawahara Y, Uetani M, Imaizumi (1993) The relationship of trabecular and cortical bone mineral density to spinal fractures. Invest Radiol 28: 573-580

2. Ito M, Hayashi K, Ishida Y, Uetani M, Yamada M, Ohki M, Nakamura T (1997) Discrimination of spinal fracture with various bone mineral measurements. Calcif Tissue Int 60:11-15

3. Ito M, Hayashi K, Yamada M, Nakamura T (1994) Vertebral measurements for assessment of osteoporosis. Br J Radiol 67: 759-763

4. Ito M, Yamada M, Hayashi K, Ohki M, Uetani M, Nakamura T (1995) Relation of early menarche to high bone mineral density. Calcif Tissue Int 57: 11-14

5. Ito M, Hayashi K, Uetani M, Yamada M, Ohki M, Nakamura T (1994) Association between anthropometric measures and spinal bone mineral density. Invest Radiol 29: 812-816

6. Ito $M$, Lang TF, Jergas M, Takada M, Nakamura T, Hayashi K, Genant HK (1997) Spinal trabecular bone loss and fracture in American and Japanese women. Calcif Tissue Int 61: 123-128

7. Ito M, Hayashi K, Yamada M, Uetani M, Nakamura T: Relationship of osteophytes to bone mineral density and spinal fracture in men. Radiology 189: 497-502, 1993

8. Engelke K, Adams JE, Armbrecht G, Augat P, Bogado CE, Bouxsein ML, Felsenberg D, Ito M, Prevrhal S, Hans DB, Lewiecki EM (2008) Clinical use of quantitative computed tomography and peripheral quantitative computed tomography in the management of osteoporosis in adults: The 2007 ISCD official positions. J Clin Densit 11: $123-162$

9. Tsurusaki K, Ito M, Hayashi K (2000) Differential effects of menopause and metabolic disease on trabecular and cortical bone assessed by peripheral quantitative computed tomography(pQCT). Br J Radiol 73: 14-22

10. Ito M, Nakamura T, Tsurusaki K, Uetani M, Hayashi K (1999) Effects of menopause on age-dependent bone loss in the axial and appendicular skeletons in healthy Japanese women. Osteoporos Int 10: 377-383

11. Ito M, Matsumoto T, Enomoto H, Tsurusaki K, Hayashi K (1999) Effect of nonweight bearing on tibial bone density measured by QCT in patients with hip surgery. J Bone Miner Metab 17: 45-50

12. Ito M, Nakamura T, Ikeda S, Tahara $Y$, Hashmi R, Tsurusaki K, Uetani M, Hayashi K (2001) Effects of lifetime volleyball exercise on bone mineral densities in lumbar spine, calcaneus and tibia for pre-, periand postmenopausal women. Osteoporos Int 12: 104-111

13. Hildebrand T, Ruegsegger P (1997) Quantification of Bone 
Microarchitecture with the Structure Model Index. In Computer Methods in Biomechanics and Biomedical Engineering, Vol. 1, pp15-23

14. Harrigan TP, Mann RW (1984) Characterization of microstructural anisotropy in orthopedic materials using a second rank tensor. J Mater Sci 19:761-767

15. Odgaard A, Gundersen HJ (1993) Quantification of connectivity in cancellous bone, with special emphasis on 3-D reconstructions. Bone $14: 173-82$

16. Ito M, Nishida A, Nakamura T, Uetani M, Hayashi K (2002) Differences of three-dimensional trabecular microstructure in osteopenic rat models caused by ovariectomy and neurectomy. Bone 30: 594-598

17. Ikeda S, Morishita Y, Tsutsumi H, Ito M, Shiraishi A, Arita S, Akahoshi S, Narusawa K, Nakamura T (2003) Reductions in bone turnover, mineral, and structure associated with mechanical properties of lumbar vertebra and femur in glucocorticoid-treated growing minipigs. Bone 33: 779-787

18. Ito M, Nakamura T, Matsumoto T, Tsurusaki K, Hayashi K (1998) Analysis of trabecular microarchitecture of human iliac bone using microcomputed tomography in patients with hip arthrosis with or without vertebral fracture. Bone 23: 163-169

19. Ikeda S, Tsurukami H, Ito M, Sakai A, Sakata T, Nishida S, Takeda S, Shiraishi A, Nakamura T (2001) Effect of trabecular bone contour on ultimate strength of lumbar vertebra after bilateral ovariectomy in rats. Bone 28: 625-633

20. Ito M, Nishida A, Aoyagi K, Uetani M, Hayashi K, Kawase M (2005) Effects of risedronate on trabecular microstructure and biomechanical properties in ovariectomized rat tibia. Osteoporos Int 16:1042-1048

21. Onoe Y, Miyaura C, Ito M, Ohta H, Nozawa S, Suda T (2000) Comparative effects of estrogen and raloxifene on $B$ lymphopoiesis and bone loss induced by sex steriod deficiency in mice. J Bone Miner Res 15: 541-549

22. Shiraishi A, Higashi S, Masaki T, Saito M, Ito M, Ikeda S, Nakamura $T$ (2002) A comparison of alfacalcidol and menatetrenone for the treatment of bone loss in an ovariectomized rat model of osteoporosis. Calcif Tissue Int 71: 69-79

23. Komatsubara S, Mori S, Mashiba T, Ito M, Li J, Kaji Y, Akiyama T, Miyamoto K, Cao Y, Kawanishi J, Norimatsu H (2003) Long-term treatment of incadronate disodium accumulates microdamage but improves the trabecular bone microarchitecture in dog vertebra. J Bone Miner Res 18: 512-520

24. Arita S, Ikeda S, Sakai A, Okimoto N, Akahoshi S, Nagashima M, Nishida A, Ito M, Nakamura T (2004) Human parathyroid hormone (1-34) increases mass and structure of the cortical shell, with 
resultant increase in lumbar bone strength, in ovariectomized rats. J Bone Miner Metab 22: 530-540

25. Miki T, Nakatsuka K, Naka H, Masaki H, Imanishi Y, Ito M, Inaba M, Morii $\mathrm{H}$, Nishizawa Y (2004) Effect and safety of intermittent weekly administration of human parathyroid hormone 1-34 in patients with primary osteoporosis evaluated by histomorphometry and microstructural analysis of iliac trabecular bone before and after 1 year of treatment. J Bone Miner Metab 22: 569-576

26. Shiraishi A, Ito M, Hayakawa N, Kubota N, Kubodera N, Ogata E (2006) Calcium supplementation does not reproduce the pharmacological efficacy of alfacalcidol for the treatment of osteoporosis in rats. Calcif Tissue Int 78: 152-161

27. Ito M, Nakayama K, Konaka A, Sakata K, Ikeda K, Maruyama T (2006) Effects of prostaglandin EP4 agonist, ONO-4819, and risedronate on trabecular microstructure and bone strength in mature ovariectomized rats. Bone 39: 453-459

28. Mori $H$, Tanaka M, Kayasuga R, Masuda T, Ochi $Y$, Yamada $H$, Kishikawa K, Ito M, Nakamura T (2008) Minodronic acid (ONO-5920/YM529) prevents decrease in bone mineral density and bone strength, and improves bone microarchitecture in ovariectomized cynomolgus monkeys. Bone 43: 840-848

29. Hishiya A, Ito M, Aburatani $H$, Motoyama N, Ikeda K, Watanabe $K$ (2005) Ataxia telangiectasia mutated (Atm) knockout mice as a model of osteopenia due to impaired bone formation. Bone 37: 497-503

30. Hiramatsu K, Asaba Y, Takeshita S, Nimura Y, Tatsumi S, Katagiri N, Niida S, Nakajima T, Tanaka S, Ito M, Karsenty G, Ikeda K (2007) Overexpression of $Y$-glutamyltransferase in transgenic mice accelerates bone resorption and causes osteoporosis. Endocrinology 148: $2708-2715$

31. Tatsumi S, Ishii K, Amizuka N, Li M, Kobayashi T, Kohno K, Ito M, Takeshita S, Ikeda K (2007) Targeted ablation of osteocytes induces osteoporosis with defective mechanotransduction. Cell Metabolism 5: 464-475

32. Ishii $K$, Fumoto $T$, Iwai $K$, Takeshita $S$, Ito $M$, Shimohata $N$, Aburatani H, Taketani S, Lelliott CJ, Vidal-Puig A, Ikeda K (2009) Coordination of PGC- $1 \beta$ and iron uptake in mitochondrial biogenesis and osteoclast activation. Nature Medicine 15: 259-266

33. Ito $M$, Ejiri $S$, Jinnai $H$, Kono J, Ikeda $S$, Nishida A, Uesugi K, Yagi N, Tanaka M, Hayashi K (2003) Bone structure and mineralization demonstrated using synchrotron radiation computed tomography (SR-CR) in animal models: preliminary findings. J Bone Miner Metab 21: 287-293

34. Chappard C, Peyrin F, Bonnassie A, Lemineur G, Brunet-Imbault B, Lespessailles E, Benhamou C-L (2006) Subchondral bone 
micro-architectural alterations in osteoarthritis: a synchrotron micro-computed tomography study. Osteoarthr Cartilage 14:215-223 35. Roschger P, Manjubala I, Zoeger N, Meirer F, Simon R, Li C, Fratzl-Zelman N, Misof BM, Paschalis EP, Streli C, Fratzl P, Klaushofer $\mathrm{K}$ (2010) Bone material quality in transiliac bone biopsies of postmenopausal osteoporotic women after 3 years of strontium ranelate treatment. J Bone Miner Res 25: 891-900

36. Andrews JC, Almeida E, van der Meulen MC, Alwood JS, Lee C. Liu Y. Chen J, Meirer F, Feser M, Gelb J, Rudati J, Tkachuk A, Yun W, Pianetta $P(2010)$ Nanoscale $X$-ray microscopic imaging of mammalian mineralized tissue. Microsc Microanal 16: 327-336

37. Voide R, Schneider P, Stauber M, Wyss P, Stampanoni M, Sennhauser U, van Lenthe GH, Muller R (2009) Time-lapsed assessment of microcrack initiation and propagation in murine cortical bone at submicrometer resolution. Bone 45: 164-173

38. Krug R, Banerjee S, Han ET, Newitt DC, Link TM, Majumdar S (2005) Feasibility of in vivo structural analysis of high-resolution magnetic resonance images of the proximal femur. Osteoporosis Int 16:1307-1314

39. Ladinsky GA, Vasilic B, Popescu AM, Wald M, Zemel BS, Snyder PJ, Loh L, Song HK, Saha PK, Wright AC, Wehrli FW (2008) Trabecular structure quantified with the MRI-based virtual bone biopsy in postmenopausal women contributes to vertebral deformity burden independent of areal vertebral BMD. J Bone Miner Res 23: 64-74

40. Chesnut CH, Majumdar S, Newitt DC, Shields A, Pelt JV, Laschansky E, Azria M, Kriegman A, Olson M, Eriksen EF, Mindeholm L (2005) Effects of salmon calcitonin on trabecular microarchitecture as determined by magnetic resonance imaging: results from the QUEST study. J Bone Miner Res 20: 1548-1561

41. Boutroy S, Bouxsein ML, Munoz F, Delmas PD (2005) In vivo assessment of trabecular bone microarchitecture by high-resolution peripheral quantitative computed tomography. J Clin Endocrinol Metab 90:6508-6515

42. Khosla S, Riggs BL, Atkinson EJ, Oberg AL, McDaniel LJ, Holets M, Peterson JM, Melton LJ (2005) Effects of sex and age on bone microstructure at the ultradistal radius: a population-based noninvasive in vivo assessment. J Bone Miner Res 21:124-131

43. Rizzoli R, Laroche M, Kreig MA, Frieling I, Thomas T, Delmas P, Felsenberg $D$ (2010) Strontium ranelate and alendronate have differing effects on distal tibial bone microstructure in women with osteoporosis. Rheumatol Int 30:1341-1348

44. Macdonald HM, Nishiyama KK, Hanley DA, Boyd SK (2010) Changes in trabecular and cortical bone microarchitecture at peripheral sites associated with 18 months of teriparatide therapy in postmenopausal women with osteoporosis. Osteoporos Int [Epub ahead of print] 
45. Ito M, Ohki M, Hayashi K, Yamada M, Uetani M, Nakamura T (1995) Trabecular texture analysis of CT images in the relationship with spinal fracture. Radiology 194:55-59

46. Ito M, Ohki M, Hayashi K, Yamada M, Uetani M, Nakamura T (1997) Relationship of spinal fracture to bone density, textural, and anthropometric parameters. Calcif Tissue Int 60: 240-243

47. Ito M, Ikeda K, Nishiguchi M, Shindo H, Uetani M, Hosoi T, Orimo H (2005) Multi-detector row CT imaging of vertebral microstructure for evaluation of fracture risk. J Bone Miner Res 20:1828-1836

48. Graeff C, Timm W, Nickelsen TN, Farrerons J, Marin F, Barker C, Gluer CC (2007) Monitoring teriparatide-associated changes in vertebral microstructure by high-resolution $\mathrm{CT}$ in vivo: results from the EUROFORS Study. J Bone Miner Res 22:1426-1433

49. Keyak JH, Rossi SA (2000) Prediction of femoral fracture load using finite element models: an examination of stress- and strain-based failure theories. J Biomech 33:209-214

50. Ito M, Nishida A, Koga A, Ikeda S, Shiraishi A, Uetani M, Hayashi K, Nakamura T (2002) Contribution of trabecular and cortical components to the mechanical properties of bone and their regulating parameters. Bone 31: 351-358

51. Keaveny TM, Donley DW, Hoffmann PF, Mitlak BH, Glass EV, Martin JAS (2007) Effects of teriparatide and alendronate on vertebral strength as assessed by finite element modeling of QCT scans in women with osteoporosis. J Bone Minr Res 22:149-157

52. Yoshikawa T, Turner $\mathrm{CH}$, Peacock M, Slemenda CW, Weaver CM, Teegarden D, Markwardt P, Burr DB (1994) Geometric structure of the femoral neck measured using dual-energy x-ray absorptiometry. J Bone Miner Res 9: 1053-1064

53. Beck TJ, Ruff CB, Warden KE, Scott WW Jr, Rao GU (1990)

Predicting femoral neck strength from bone mineral data. A structural approach. Invest Radiol 25:6-18

54. Ito M, Sone T, Fukunaga M (2010) Effect of minodronic acid hydrate on hip geometry in Japanese women with postmenopausal osteoporosis. J Bone Miner Metab 28: 334-341

55. Mayhew PM, Thomas CD, Clement JG, Loveridge N, Beck TJ, Bonfield W, Burgoyne, CJ, Reeve J (2005) Relation between age, femoral neck cortical stability, and hip fracture risk. Lancet 366:129-135

56. Ito M, Wakao N, Hida T, Matusi Y, Abe Y, Aoyagi K, Uetani M, Harada A (2010) Analysis of hip geometry by clinical CT for the assessment of hip fracture risk in elderly Japanese women. Bone 46: 453-457

57. Ito M, Nakata T, Nishida A, Uetani M (2010) Age-related changes in bone density, geometry and biomechanical properties of the proximal femur: CT-based 3D hip structure analysis in normal postmenopausal women. Bone (online first) 
58. Bessho M, Ohnishi I, Okazaki H, Sato W, Kominami H, Matsunaga S, Nakamura K (2004) Prediction of the strength and fracture location of the femoral neck by CT-based finite-element method: a preliminary study on patients with hip fracture. J Orthop Sci 9: 545-550 
Figure legends

structure model index degree of anisotropy

(SMI)
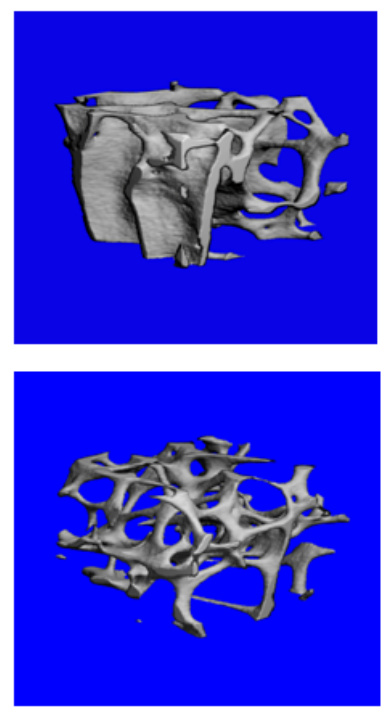

$(D A)$
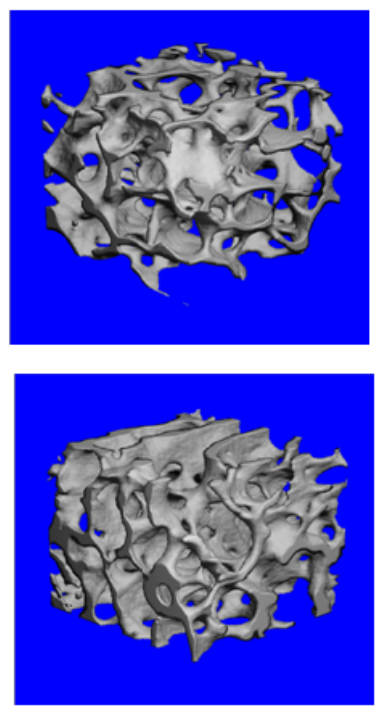

Connectivity density
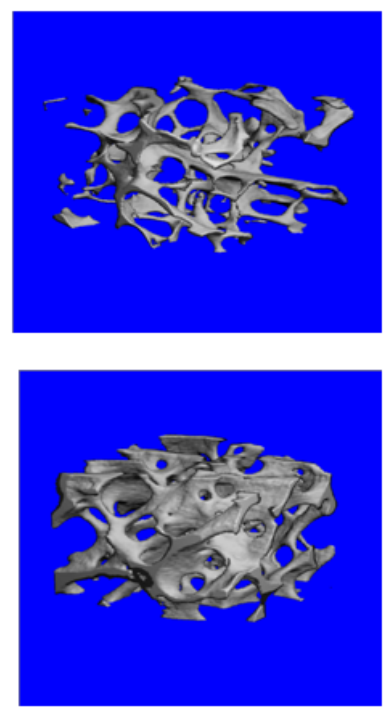

Figure 1 Non-metric parameters of the 3D microstructure Representative bone samples with different trabecular network patterns are shown (compare the upper and lower panels). Structure model index (SMI) used to quantify the shape of the trabeculae: plate-rod characteristics of the structure.

Degree of anisotropy (DA) used to quantify the orientation of the trabeculae: isotropic versus anisotropic structure.

Connectivity density used to quantify trabecular connectivity: poor and good connectivity. 


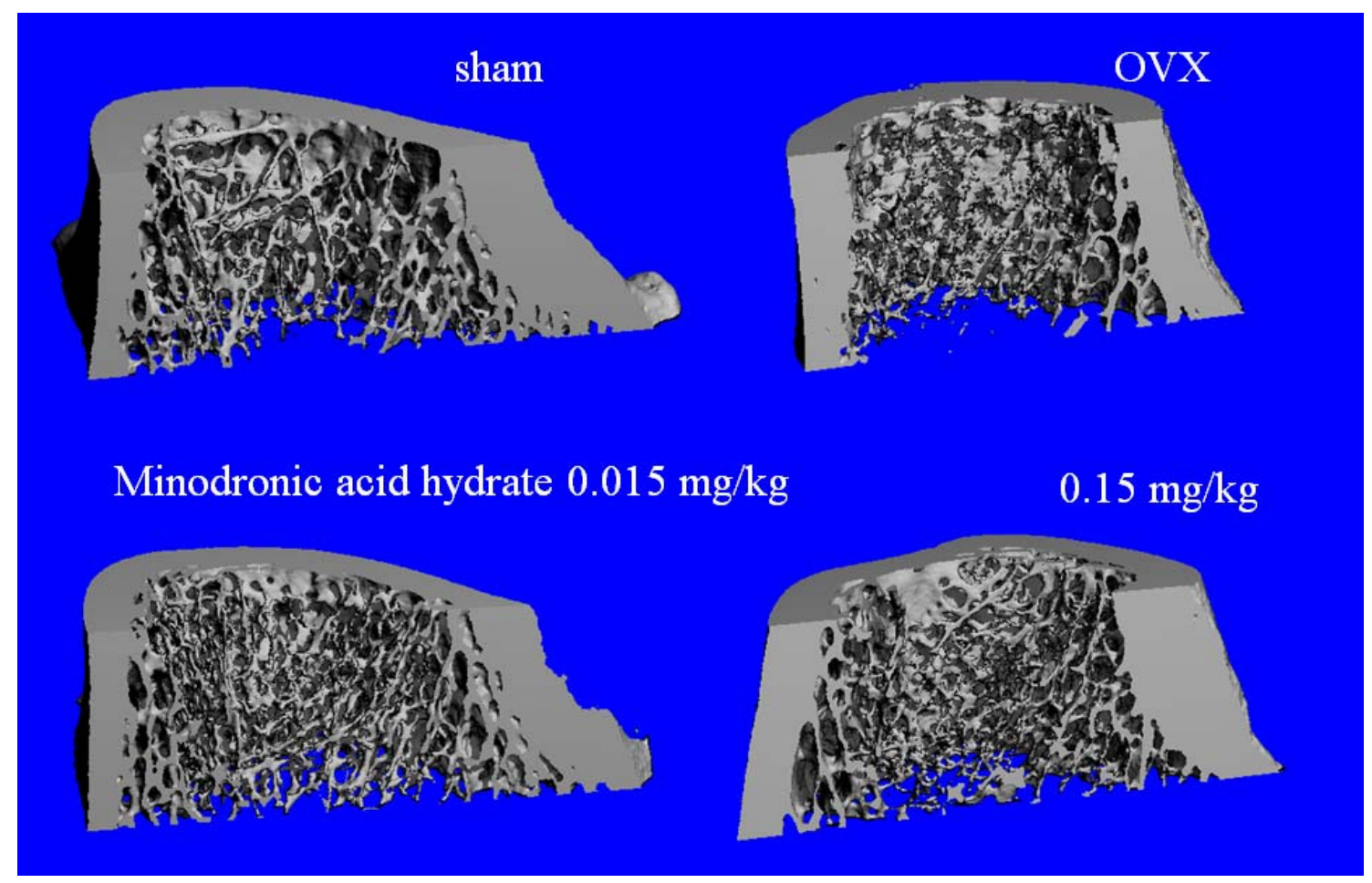

Figure 2 3D micro-CT images of the distal femur of ovariectomized cynomolgus monkeys

Results from sham, OVX and groups treated with low and high doses of minodronic acid are shown. 

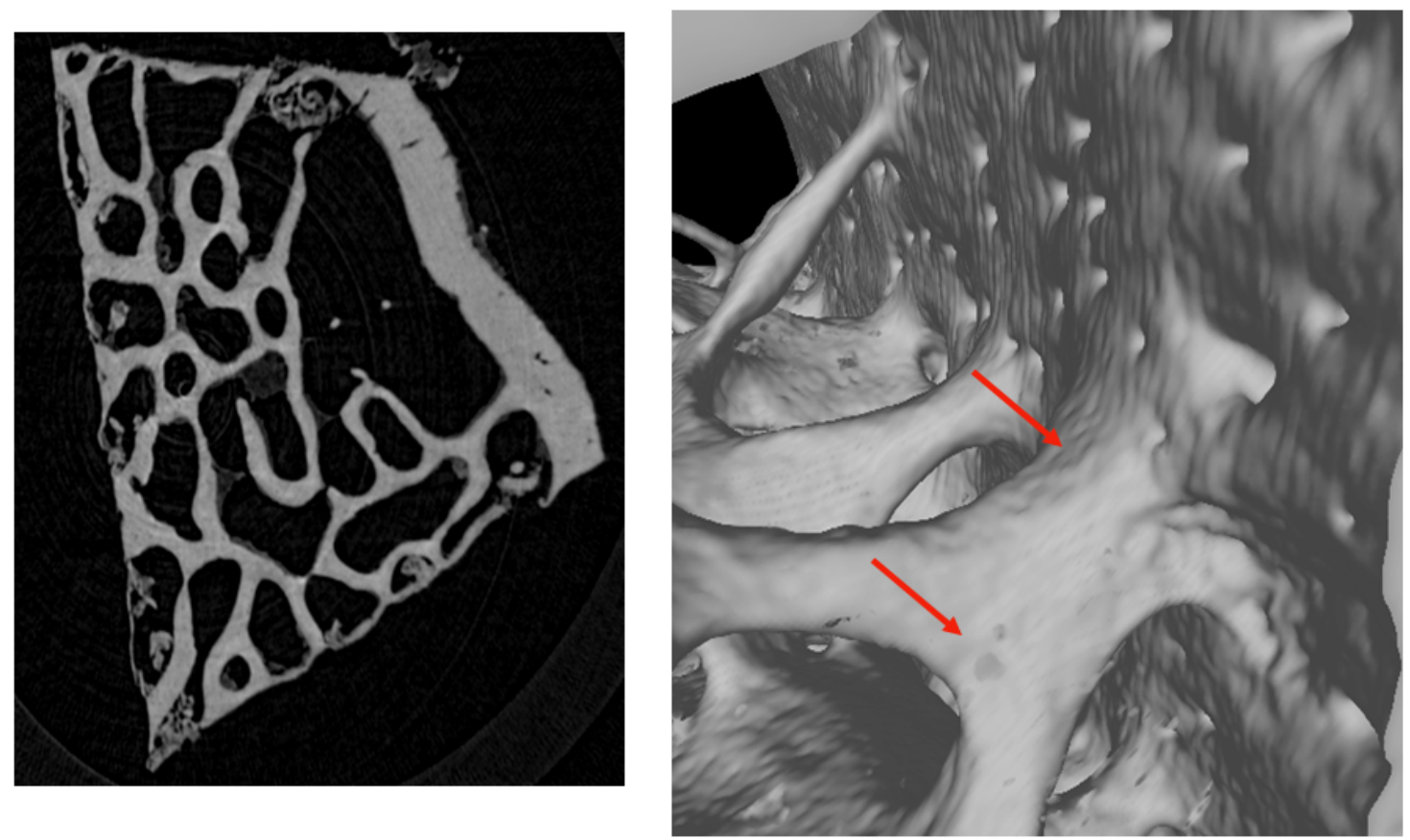

Figure 3 Synchrotron CT image of rat vertebra (2D and 3D image) 

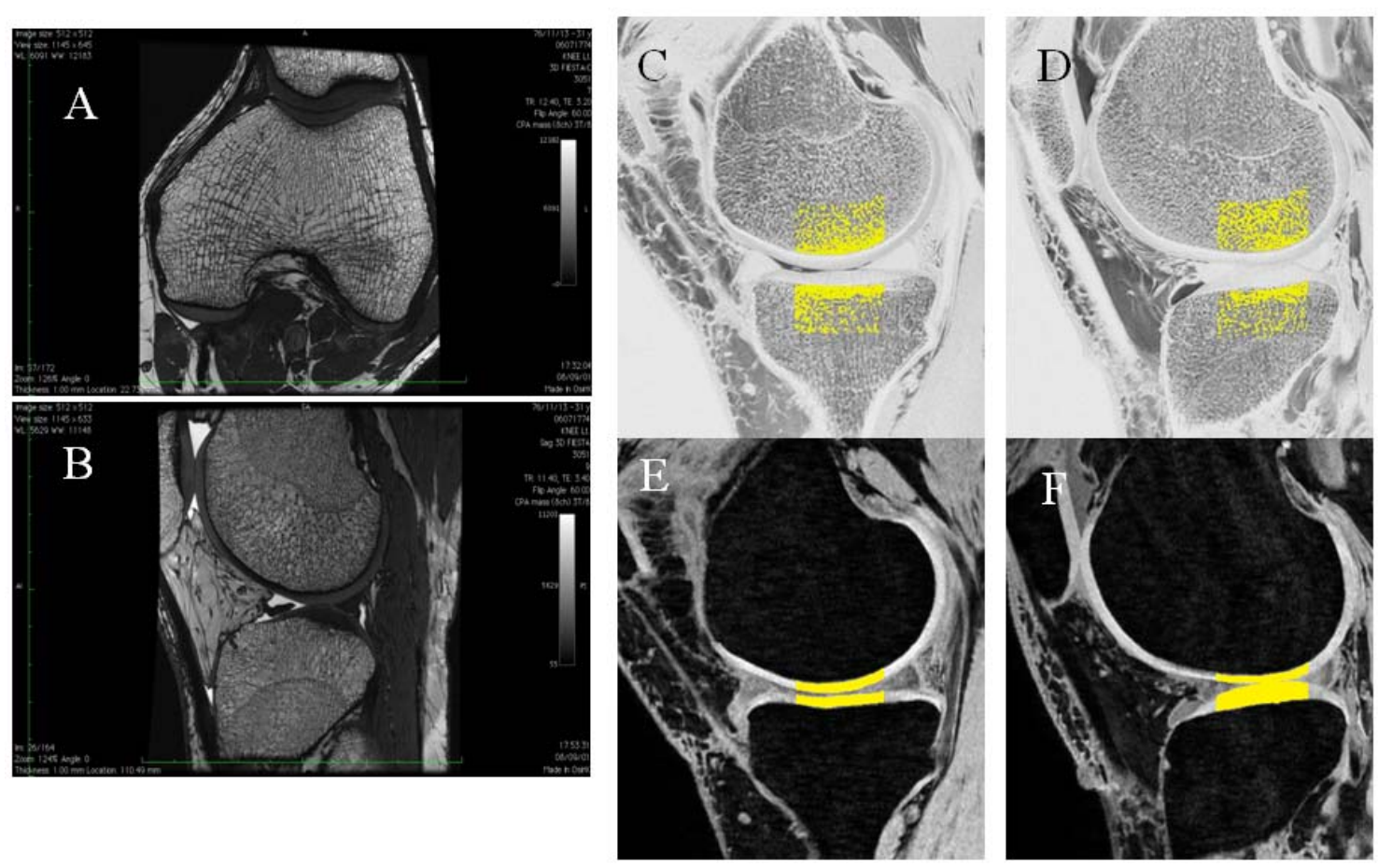

Figure $4 \mathrm{MR}$ images of the knee joint

$A, B$ : trabecular structure obtained using FIESTA-c: axial $(A)$ and sagittal (B) sections of the knee joint.

C,D: black-white reversed FIESTA-C images of the sagittal section ( $C$ : medial, D: lateral condyle).

$\mathrm{E}, \mathrm{F}$ : Analysis of articular cartilage ( $\mathrm{E}$ : medial, F: lateral condyle). 

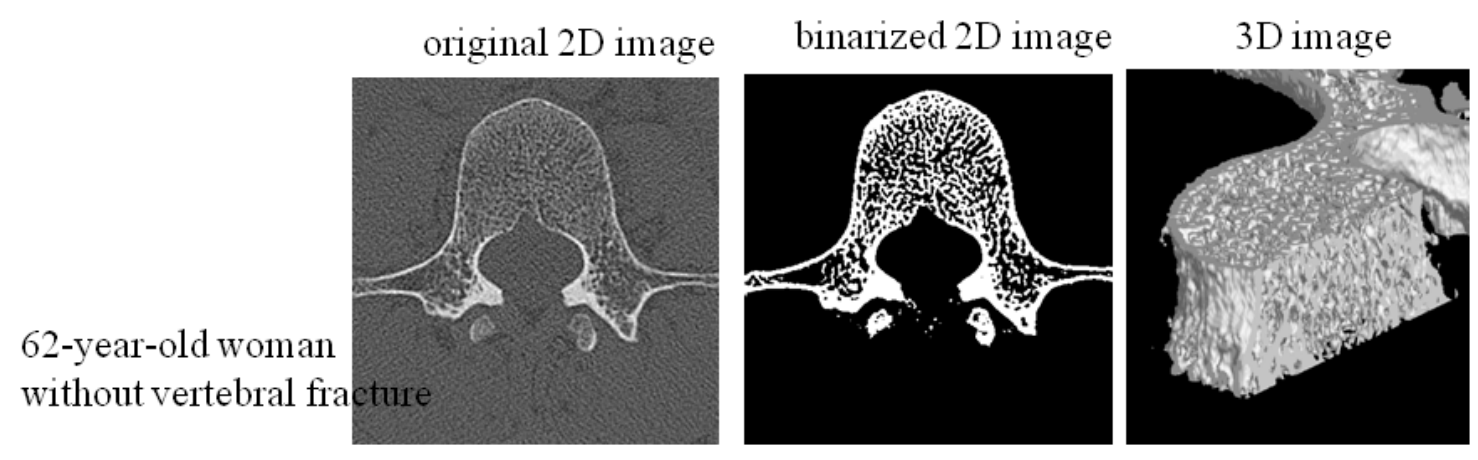

62-year-old woman
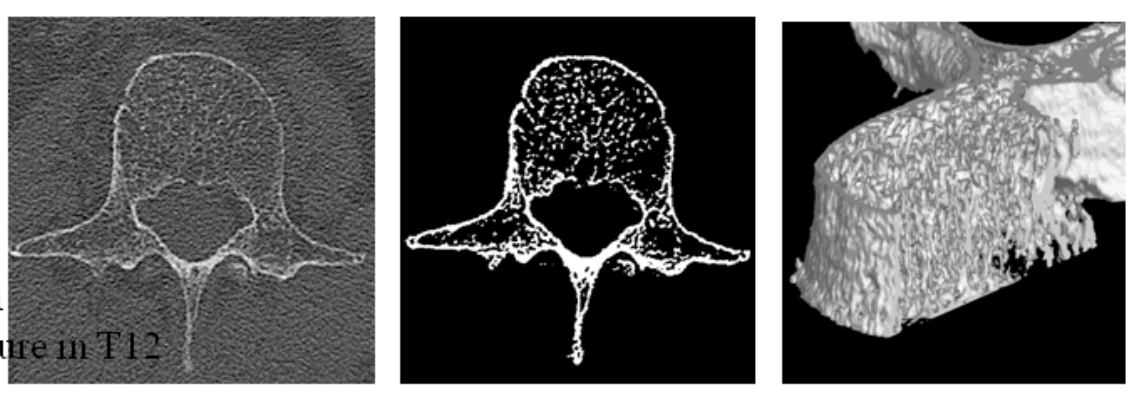

Figure5 Human vertebral trabecular network using MDCT

The original 2D (left column), binalized 2D (middle column), and 3D reconstructed (right column) CT images are shown.

Upper: L3 from a 62-year-old woman without vertebral fracture, Lower: L3 from a 62-year-old woman with a vertebral fracture in Th12. High-resolution 3D images reveal marked osteoporotic changes in the woman with the vertebral fracture. 

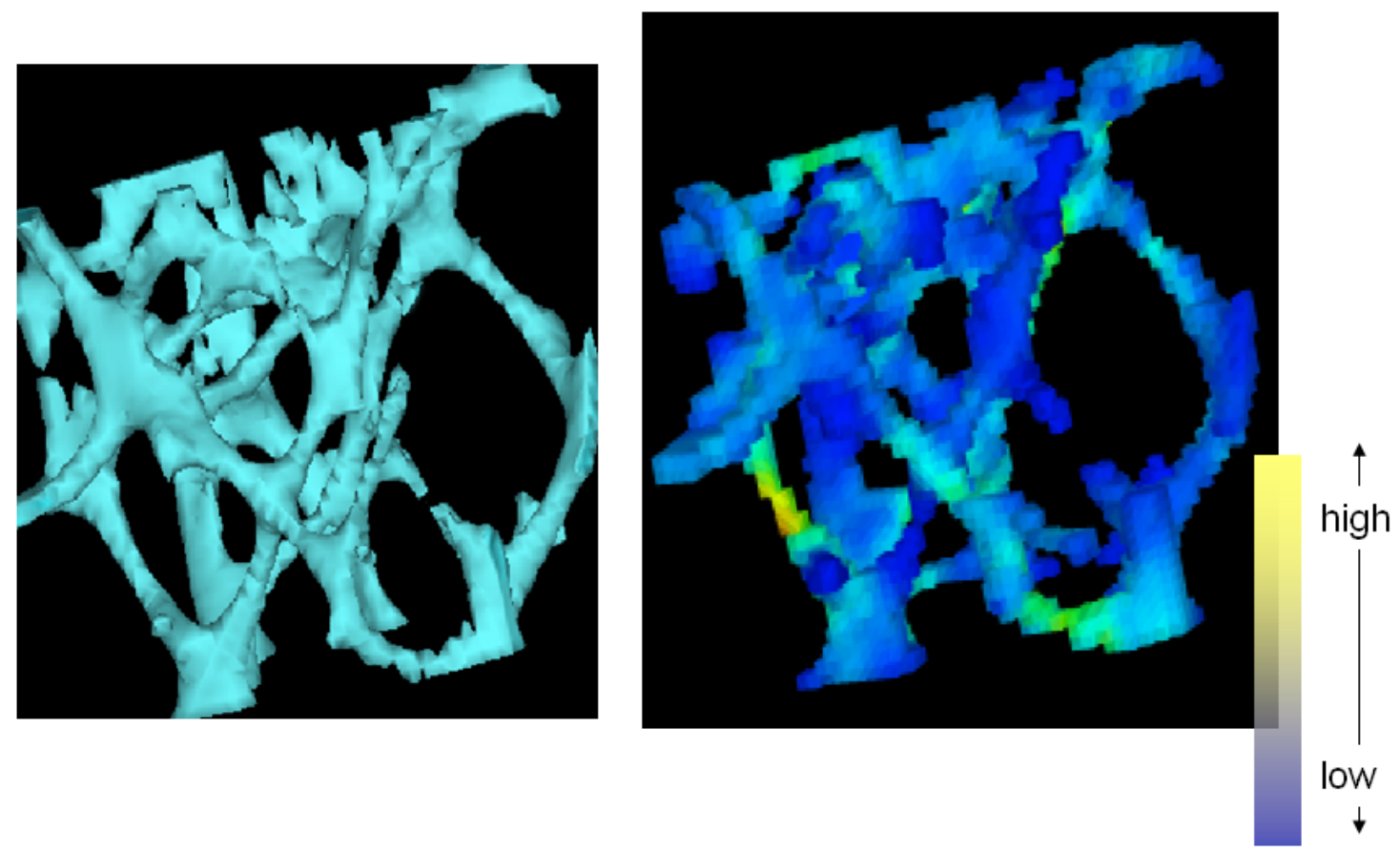

Figure6 Micro-finite element model of trabecular structure Left: an original 3D trabecular structure image.

Right: stress distribution under loading from the upper side.

The thin trabecula is shown in yellow, indicating that it is highly stressed. 

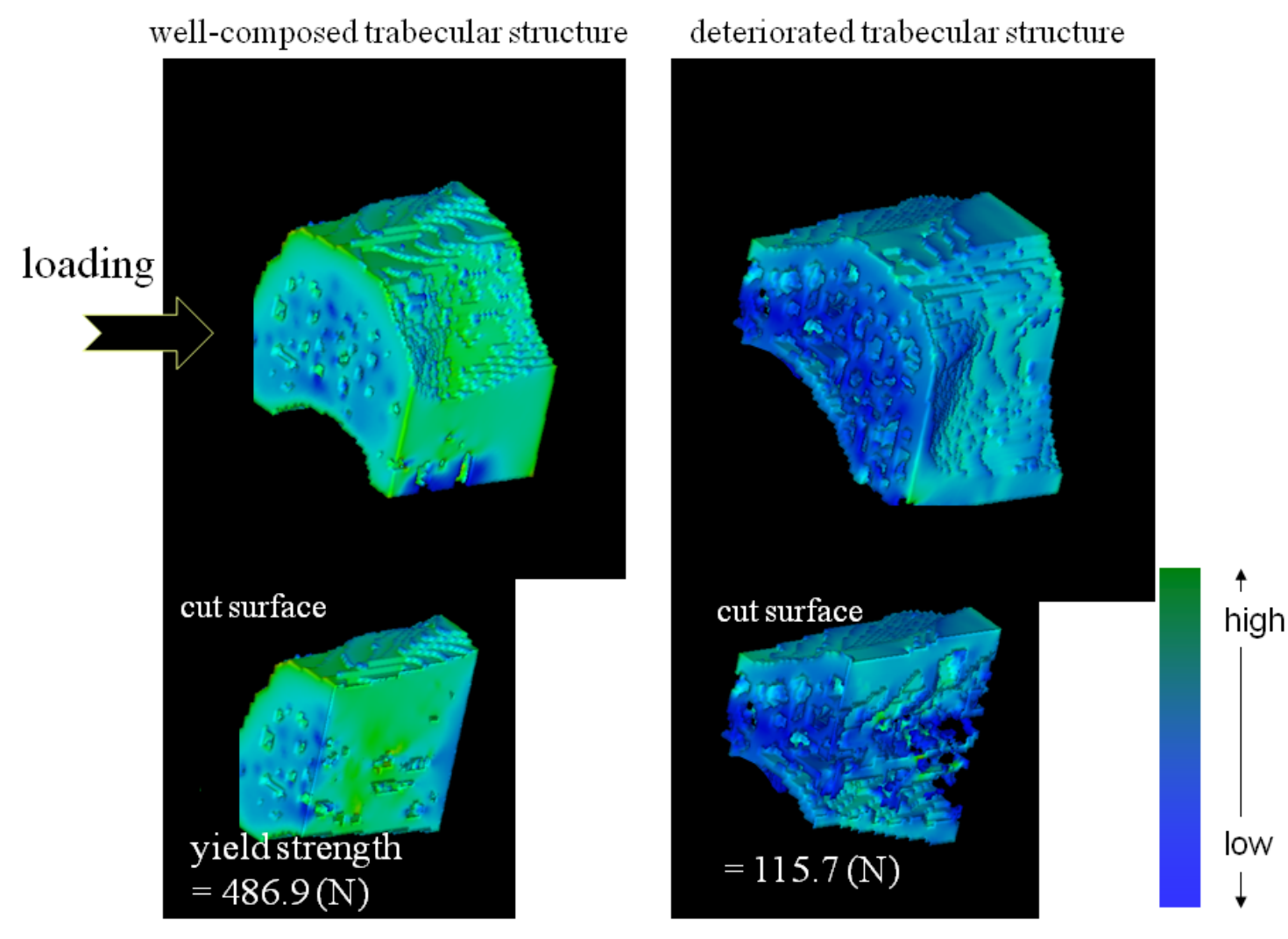

Figure7 The differences in stress distribution and yield strength Under cranio-caudal loading. The bone with the well-composed trabecular structure (left) exhibits uniform stress distribution in both the cortical and trabecular regions, while in the bone with deteriorated trabecular structure (on the right), the highest stress appears in the cortical region and there is less stress in the spongiosa. The yield strength results of the bone are $486.9(\mathrm{~N})$ and $115.7(\mathrm{~N})$, respectively. 


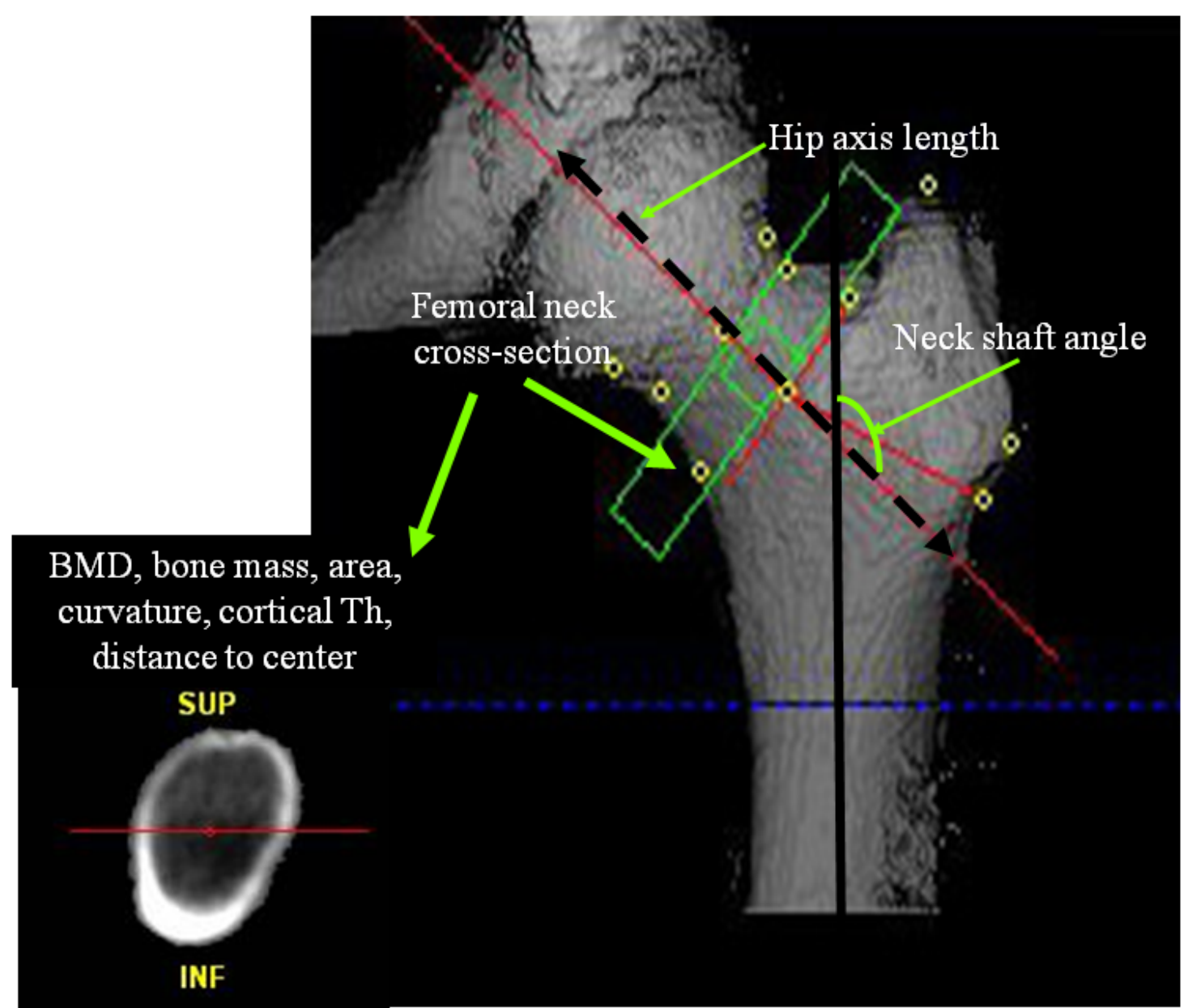

Figure8 CT-based Hip Structure Analysis

3D volumetric QCT of proximal femur measures of Hip Axis Length (HAL) and Neck Shaft Angle (NSA). Based on the axis of the femoral neck defined three-dimensionally, a cross-sectional image is obtained to allow the determination of several parameters, such as bone mass, bone area, curvature and cortical thickness, as well as biomechanical properties. 
Table - Bone imaging modalities used to quantify bone characteristics

\begin{tabular}{|c|c|c|c|c|c|c|}
\hline & \multicolumn{4}{|c|}{ Clinical Application } & \multicolumn{2}{|c|}{ Laboratory Investigation } \\
\hline & radiography & $\mathrm{DXA}$ & $\mathrm{vQCT} / \mathrm{HR}-\mathrm{CT}$ & HR-MR & micro-CT & synchrotron $\mathrm{CT}$ \\
\hline BMD measurement & $\mathrm{X}$ & $\begin{array}{c}\mathrm{O} \\
(2 \mathrm{D})\end{array}$ & $\begin{array}{c}O \\
(3 D)\end{array}$ & $X$ & O & O \\
\hline nacrostructure/hip geomtr! & $\triangle$ & O & $\mathrm{O}$ & $\mathrm{X}$ & - & - \\
\hline microstructure & $\triangle$ & $\mathrm{X}$ & $\underset{(*)}{\bigcirc}$ & $\underset{(*)}{\bigcirc}$ & O & (2) \\
\hline nanostructure & $\mathrm{X}$ & $\mathrm{X}$ & $X$ & $\mathrm{X}$ & $\begin{array}{c}\triangle-O \\
(* *)\end{array}$ & $\begin{array}{l}\text { O-@ } \\
(* *)\end{array}$ \\
\hline nineralization in micro-scal & $\mathrm{X}$ & $\mathrm{X}$ & $X$ & $X$ & $\begin{array}{c}\triangle-O \\
(* *)\end{array}$ & (2) \\
\hline cortical microstructure & $\mathrm{X}$ & $\mathrm{X}$ & X & $x$ & $\begin{array}{c}\triangle-O \\
(* *)\end{array}$ & (2) \\
\hline $\begin{array}{l}\text { Application for finite } \\
\text { element model }\end{array}$ & $\mathrm{X}$ & $\mathrm{X}$ & O & $\Delta$ & $\underset{(* * *)}{\bigcirc}$ & $\underset{(* * *)}{\bigcirc}$ \\
\hline
\end{tabular}

*: limited to apparent values, **: depending on supplied resolution, ***: small region, but at micro-scale DXA:dual X-ray absorptiometry, vQCT: volumetric quantitative computed tomography, HR-CT: high-resolution CT, HR-MR: high-resolution magnetic resonance, BMD: bone mineral density 